\title{
Putting the brain back together
}

$I^{\prime}$ $\mathrm{n}$ this age of information overload, neuroscience offers no safe haven. Thomson Scientific's Journal Citation Reports indexes 344 neuroscience journals, and there are roughly 40,000 neuroscience papers in PubMed each year. For scientists-who already juggle writing grants, reviewing papers, sitting on committees and running a lab-keeping up with mounting data in one's own field is daunting; maintaining perspective across fields is practically impossible. However, continued progress toward understanding the brain and its disorders requires that this body of information be adequately synthesized.

Larry Swanson of the University of Southern California notes that, despite a long history and large literature of neuroanatomical research, our understanding of the basic organizing principles of the mammalian brain remains uncertain ${ }^{1}$. This uncertainty is exacerbated by the lack of precise definitions for alternative terms for brain parts and their relationships. The state of neuroanatomy exemplifies the lack of integration in neuroscience research, and it may also contribute more broadly to perpetuating the problem. Without an architectural framework for anatomical connectivity, we lack a clear context for tying together other neuroscientific data.

We do not have enough data to answer many fundamental questions about brain function. Nevertheless, when data are generated faster than we can understand them, we risk leaving inconsistencies and errors unchecked, straining resources for peer review and making research more difficult. Integrative approaches to critically compare and synthesize data both across and within disciplines could help by pointing out gaps in our existing knowledge. Furthermore, allowing sufficient time for reflective thought and deliberation could facilitate the development of general principles and new hypotheses to guide future research.

Traditional integrative mechanisms, like meetings and review articles, are increasingly inadequate to cope with data overload. Meeting schedules often leave little time for consolidation, and reviews are often narrow in scope and are themselves proliferating. One strategy for harnessing large amounts of data has been the development of databases and data management tools, spurred in neuroscience research by the Human Brain Project ${ }^{2}$. These resources will be vital for facilitating integration and data sharing. However, significant hurdles remain, including the need for better community participation and for ways to increase the interoperability of the growing numbers of databases, knowledge bases and other informatics resources available. (The Society for Neuroscience's Neuroscience Database Gateway now lists 113.) More importantly, data management tools assist, but cannot replace, human understanding.

Although technological advances and a growing scientific workforce have greatly accelerated the rate of data collection and publication, the current incentive structure for academic research is a major driving force behind this expansion. Competitive grantsmanship rewards new results generated from short-term research proposals, and maintaining funding depends in turn on steady production and publication of such results. In addition to continued support for the development of informatics resources, funding agencies could help avoid negative consequences of data overload by making grant mechanisms and review criteria less contingent on output from new lines of research.

To shift the emphasis toward quality rather than quantity of scientific results, funding agencies could support specific integrative initiatives, such as large-scale meta-analyses in unresolved areas or experiments to tackle particularly contentious conflicts in the existing literature. One successful example is an ongoing analysis of genetic association studies by Alzheimer disease researchers at Massachusetts General Hospital. Increasingly frustrated with confusing and conflicting reports of genes associatedor not—with late-onset Alzheimer disease, Lars Bertram and Rudy Tanzi built their own database updated regularly from the literature to keep a running score (http://www.alzgene.org). In contrast to meetings that focus on presenting new data, funding agencies and academic societies might also consider sponsoring regular meetings of think tank-like groups of scientists to discuss the state, direction and needs of their discipline or ways to bridge research between disciplines, and then share their insights with the larger community.

Simply having more time to think and interact with colleagues could foster consolidation and conceptual breakthroughs. Unfortunately for many academic researchers, such ruminating might carry the stigma of inactivity or, worse, speculation. However, science is largely a creative process, and the minds of scientists are ultimately its greatest resource. Legitimizing time for creative synthetic thought through funding might be an inexpensive way to shift the current incentive structure. One model might be the OPUS (Opportunities for Promoting Understanding through Synthesis) award created by the Division of Environmental Biology at the National Science Foundation to provide partial salary support to mid-senior level scientists to undertake a critical synthesis of their own work. The Howard Hughes Medical Institute has long funded scientists, rather than individual projects, and their new Janelia Farm Research Campus will further free researchers from grant-writing, teaching and administrative duties. Set to open this year with a major focus on deciphering neuronal circuits, Janelia will also promote integration by supporting longterm collaborative research in an environment designed to encourage informal interactions.

Not all of neuroscience research needs to turn to integrative efforts: even a modest shift in these directions could benefit the entire research community. We have become adept at taking the brain apart and examining all the pieces; we may still need to learn how best to put it all back together again. Changes occur most effectively through training the next generation, and yet, as Richard Morris at the University of Edinburgh says, "The first thing we want to do when we get a new student or postdoc is to get them in the lab doing experiments. No one ever tells them to just go to the library and think." Perhaps we should.

1. Bota, M., Dong H.-W. \& Swanson, L.W. Nat. Neurosci. 6, 795-799 (2003)

2. Martone, M.E., Gupta, A. \& Ellisman, M.H. Nat. Neurosci. 7, 467-472 (2004).

View more background material on Connotea at http:// connotea.org/user/NatNeurosci/tag/editorial200604. 